\title{
STABILITY OF A TANDEM OF QUEUEING SYSTEMS WITH BERNOULLI NONINSTANTANEOUS TRANSFER OF CUSTOMERS
}

UDC 519.21

\author{
A. V. ZORIN
}

\begin{abstract}
A tandem of queueing systems with cyclic control algorithm and with independent conflict input flows is considered. The customers served in the first queueing system are transferred to the second system with random speeds. A cybernetic approach is used to construct a mathematical model in terms of a multidimensional denumerable Markov chain. The classification of the states of this Markov chain is carried out, and a sufficient condition for the existence of the stationary distribution is obtained.
\end{abstract}

\section{The Setting of the PRoblem}

Consider two queueing systems serving the customers under the following assumptions. There are four independent conflict input flows, two flows for each of the systems. Each of these flows is a nonordinary Poisson process. The flows are conflicting in the sense that customers arriving from different sources cannot be served simultaneously in the same queueing system. This means that the problem cannot be reduced to a corresponding problem with fewer input flows by merging the flows together. The customers coming from each flow are placed in a special buffer with an unbounded capacity. The service time of an arbitrary customer is random and service times of different customers are independent random variables with unknown probability distributions. Each system may serve several customers during the same working period. The service algorithm of conflict flows in each of the two systems belongs to the class of cyclic algorithms. We assume that the durations of working periods are commensurable constants.

At the moment 0 , the service starts in both systems. After a service is completed in the first system for a customer coming from the first flow of the first system, he is transferred to the second system. We assume that the transfer is not instant. We further assume that the speed of the transfer for each of the customers is random and its probability distribution is unknown. Moreover, the speeds of transfer of different customers are varying with time and have different probability distributions. We agree that the transfer of every customer from the first system to the second system is completed with a known probability during a working period. Respectively, the transfer is not completed during a working period and will be continued during the next one with the complementary probability.

2010 Mathematics Subject Classification. Primary 60K25, 90B22.

Key words and phrases. Controlled queueing system, cybernetic approach, nonlocal description of the input flow, Markov chain, stationary distribution.

This work is supported by the State Scientific Research Project \# 0120.0602598 "The analysis of discrete control queueing systems and systems for the evaluation of Boolean functions" at the Lobachevskir State University of Nizhniǔ Novgorod. 
The customers from the first system are placed in the queue together with the first input flow of the second system. This means that the input flow for the second queueing system has a complicated probability structure.

Finally, the customers coming from the second input flow of the first system as well as all the customers in the second system leave the tandem of queueing systems after the service.

An example of a tandem of queueing systems with a cyclic control algorithm in each of the two systems is represented by a chain of two crossroads (see Figure 1) with input traffic flows $\Pi_{1}, \Pi_{2}, \ldots, \Pi_{5}$. The flows $\Pi_{1}$ and $\Pi_{4}$ are conflicting as well as the superposition of the flows $\Pi_{2}$ and $\Pi_{3}$ is conflicting with the flow $\Pi_{5}$. The flow $\Pi_{3}$ is the output for the first crossroad.

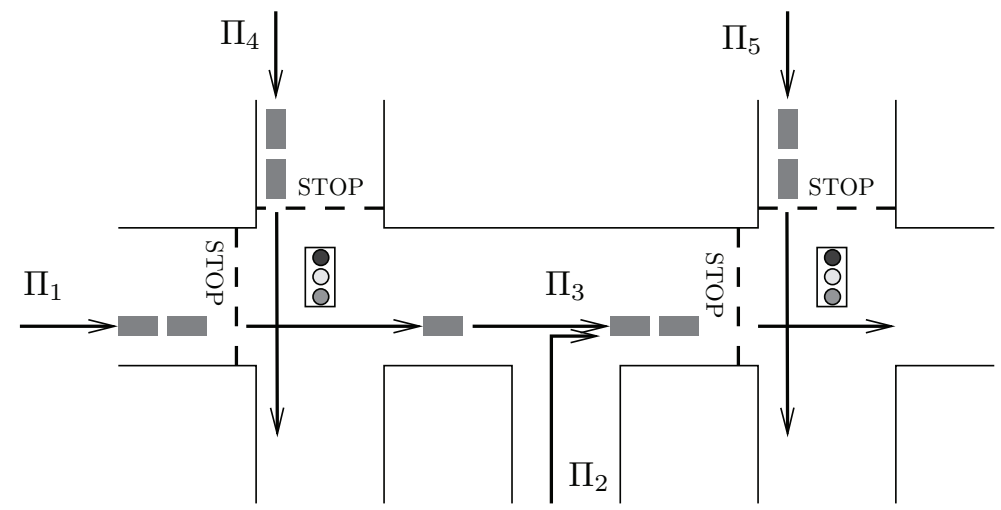

Figure 1. Tandem of crossroads

Note that the output flow $\Pi_{3}$ comes from the first system that cyclically switches from the service of the flow $\Pi_{1}$ to blocking it and vice versa. The times when the customers leave the first system depend on the service times that are dependent and have unknown and different probability distributions.

This model does not fit the classical description of the output flow in terms of a counting process $\{\eta(t) ; t \geq 0\}$ with continuous time (the same concerns any equivalent classical description). Instead, we use a nonlocal description of the flows and a cybernetic approach to construct and analyze the mathematical models of controlled queueing systems [1]- 4]. The nonlocal description of a flow of customers $\Pi$ means that a marked point process $\left\{\left(\tau_{i}^{\prime}, \eta_{i}^{\prime}, \nu_{i}^{\prime}\right) ; i=1,2, \ldots\right\}$ is given, where $\tau_{i}^{\prime}$ is the moment of observation, $\tau_{0}^{\prime}=0, \eta_{i}$ is the number of customers arriving during the interval $\left(\tau_{i-1}, \tau_{i}\right]$, and where $\nu_{i}^{\prime}$ is the mark of these customers.

The following are the three main principles of the cybernetic approach:

a) the controlled queueing system operates at discrete moments $\tau_{i}, i=0,1, \ldots$;

b) the nonlocal description of the structure of a controlled queueing system is given blockwise;

c) the block structure of a controlled queueing system and its functioning in time are studied jointly.

Every controlled queueing system has its scheme, information, coordinates, and function. The scheme of a queueing system consists of the following seven blocks:

1) external environment,

2) input poles,

3) external memory, 
4) a device processing the information in the external memory,

5) internal memory,

6) a device processing the information in the internal memory,

7) output poles.

The information in the queueing system is the set of all possible states of its blocks. A coordinate of the queueing system defines the state of a corresponding block. The function of the queueing system describes the way in which the controlled queueing system passes from one discrete time moment to another one.

In the rest of the paper we consider the input flows $\Pi_{1}, \Pi_{2}, \Pi_{3}$ only. An analogous approach can be applied to study all five input flows $\Pi_{1}, \Pi_{2}, \ldots, \Pi_{5}$.

Denote by $O_{1}$ and $O_{2}$ the buffers for the customers arriving from the flow $\Pi_{1}$ and $\Pi_{2}$, respectively. By $\mathrm{O}_{3}$, we denote the buffer for the flow of customers transferred from the first system to the second one.

The following notation is needed to define the input processes and service discipline. Let $\lambda_{j}$ denote the intensity for the bulk arrival for the flow $\Pi_{j}, j=1,2$. Further, let $p_{x}^{(j)}$ be the probability that the size of a group of customers arriving from the flow $\Pi_{j}$ is equal to $x, x=1,2, \ldots$. We assume that the following two series

$$
\sum_{x=1}^{\infty} z^{x} p_{x}^{(j)}, \quad j=1,2,
$$

converge in the circle $|z|<1+\varepsilon$ for some $\varepsilon>0$.

The duration of the working period for customers from the queue $O_{1}$ is equal to $T_{1}^{\prime}$. Similarly, $T_{2}^{\prime}$ is the duration of the working period for the flow $\Pi_{4}, T_{3}^{\prime}$ is the duration of the working period for the flows $\Pi_{2}$ and $\Pi_{3}$, and $T_{4}^{\prime}$ is the duration of the working period for the flow $\Pi_{5}$.

To fix a discrete time scale consider the moments $\tau_{1}, \tau_{2}, \ldots$ when at least one of the servers changes its state; we also put $\tau_{0}=0$. We treat two servers as a new server with $n<\infty$ states $\Gamma^{(1)}, \Gamma^{(2)}, \ldots, \Gamma^{(n)}$ and with sojourn times $T_{1}, T_{2}, \ldots, T_{n}$. The number $n$ and sojourn times are chosen according to $T_{1}^{\prime}, T_{2}^{\prime}, T_{3}^{\prime}, T_{4}^{\prime}$ and also to the initial states of servers for both queueing systems. In the state $\Gamma^{(r)}$, either none of the customers arriving from the queues $O_{1}, O_{2}$ is served (the state of type I), or only the customers from the queue $O_{1}$ are served (the state of type II), or only the customers from the queue $O_{2}$ are served (the state of type III), or customers from both queues $O_{1}$ and $O_{2}$ are served (the state of type IV). For example, let $T_{1}^{\prime}=5, T_{2}^{\prime}=4, T_{3}^{\prime}=3, T_{4}^{\prime}=3$ and let the service start at a moment $\tau_{0}$ for flows $\Pi_{1}$ and $\Pi_{5}$. Then $n=8$ and

$$
\begin{gathered}
\tau_{1}=3, \tau_{2}=5, \tau_{3}=6, \tau_{4}=9, \tau_{5}=12, \tau_{6}=14, \tau_{7}=15, \tau_{8}=18, \tau_{9}=21, \ldots ; \\
T_{1}=3, T_{2}=2, T_{3}=1, T_{4}=3, T_{5}=3, T_{6}=2, T_{7}=1, T_{8}=3 .
\end{gathered}
$$

Thus the states $\Gamma^{(4)}$ and $\Gamma^{(7)}$ are of type I, $\Gamma^{(1)}$ and $\Gamma^{(6)}$ are of type II, $\Gamma^{(3)}$ and $\Gamma^{(8)}$ are of type III, and $\Gamma^{(2)}$ and $\Gamma^{(5)}$ are of type IV.

Finally, we assume that each customer from the queue $O_{3}$ is transferred to the queue $\mathrm{O}_{2}$ with probability $p_{r}$ independently of all other customers in the queue $\mathrm{O}_{3}$ provided the server is in the state $\Gamma^{(r)}$ during the time $T_{r}$.

Since the durations of working periods for different customers are dependent and have different probability distributions, it is convenient to describe the service process in terms of the saturation flows $\Pi_{1}^{\text {sat }}$ and $\Pi_{2}^{\text {sat }}$ instead of in terms of the distribution of the duration of the working period for an arbitrary customer [1. The saturation flow is a virtual output flow corresponding to the maximal workload of the servers and highest queue loads. The saturation flow $\Pi_{1}^{\text {sat }}$ contains 0 customers if the state of the server $\Gamma^{(r)}$ is of type I or III, while $\Pi_{1}^{\text {sat }}$ contains a nonrandom number $\ell_{r, 1}>0$ of customers if the 
state of the server $\Gamma^{(r)}$ is of type II or IV. Similarly, the saturation flow $\Pi_{2}^{\text {sat }}$ contains 0 customers if the state $\Gamma^{(r)}$ is of type I or II and contains a nonrandom number $\ell_{r, 2}>0$ of customers if $\Gamma^{(r)}$ is of type III or IV.

The scheme of a controlled queueing system is depicted in Figure 2, The scheme contains the following blocks:

1) external environment,

2) input flows $\Pi_{1}, \Pi_{2}$, and $\Pi_{3}$ (input flows of the first type) and saturation flows $\Pi_{1}^{\text {sat }}$ and $\Pi_{2}^{\text {sat }}$ (input flows of the second type),

3) buffers $O_{1}, O_{2}$, and $O_{3}$ (external memory),

4) devices $\beta_{1}, \beta_{2}$, and $\beta_{3}$ processing the information (the discipline of service in queues) in the external memory,

5) server (internal memory),

6) graph of changes of states of the server (a device processing the information in the internal memory),

7) output flows $\Pi_{1}^{\text {out }}$ and $\Pi_{2}^{\text {out }}$ (output poles).

The set of all possible states of the random environment, input flows, buffers, server, saturation flows, and output flows describes the information in the controlled queueing system. The numbers corresponding to the states of the external environment, input flows, buffers, devices realizing the discipline in the queues, server, saturation flows, and output flows are the coordinates of the queueing system. The function of the system is to serve customers according to a cyclic algorithm when the order and the working period are fixed.

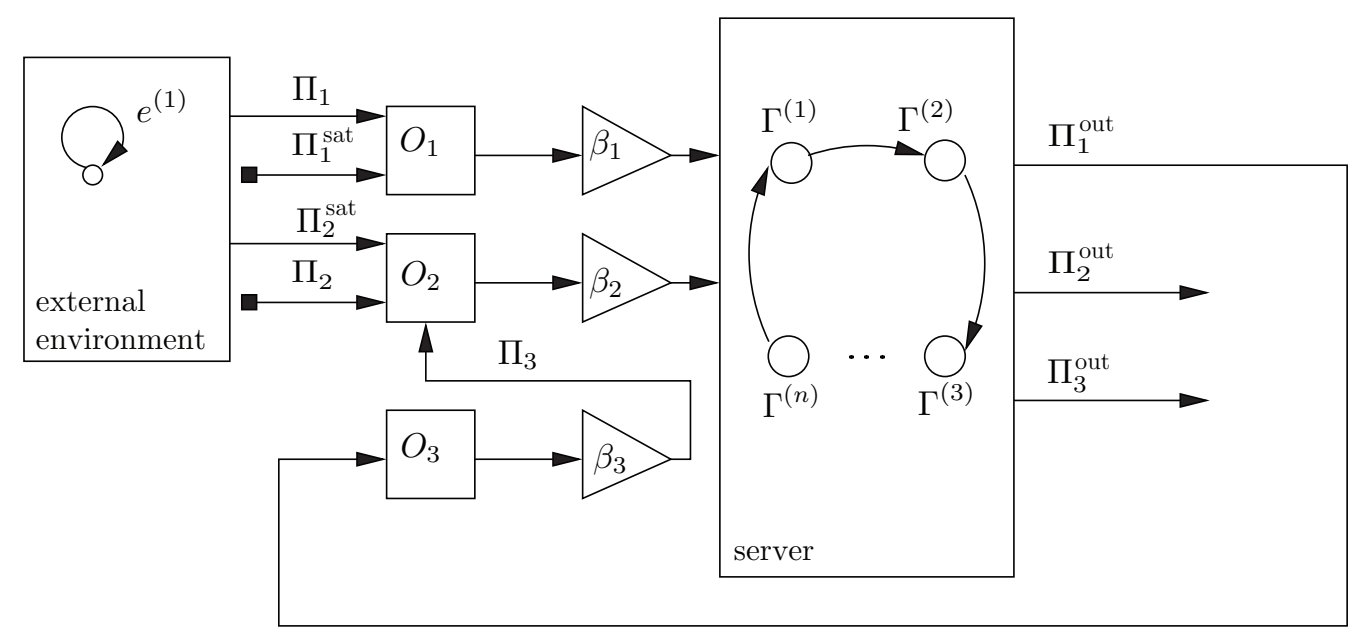

FIgURE 2. The scheme of the tandem of queueing systems (with cyclic service algorithm when the order and the working period are fixed) as a controlled queueing system

\section{Mathematical Model}

We assume that all random variables and other random elements are defined on a probability space $(\Omega, \mathfrak{F}, \mathrm{P})$. The sequence $\tau_{0}=0, \tau_{1}, \ldots, \tau_{i}, \ldots$ of observation moments is defined above. By $\Gamma_{i}$, we denote the state of the server at the moment $\tau_{i}$ and let $\kappa_{s, i}$ be the number of customers in the buffer $O_{s}$ for $s=1,2$, and 3 at the moment $\tau_{i}$. Further let $\eta_{s, i}$ and $\xi_{j, i}$ be the number of customers arriving from the flows $\Pi_{s}$ and $\Pi_{j}^{\text {sat }}$, 
respectively, during the interval $\left(\tau_{i}, \tau_{i+1}\right]$, and let $\bar{\xi}_{1, i}$ be the number of customers from the flow $\Pi_{1}^{\text {out }}$ whose service is completed during the interval $\left(\tau_{i}, \tau_{i+1}\right]$.

For $r, 1 \leq r<n$, let $r \oplus 1=r+1$ and $n \oplus 1=1$. Set $\Gamma=\left\{\Gamma^{(1)}, \Gamma^{(2)}, \ldots, \Gamma^{(n)}\right\}$ and introduce the mapping $u(\cdot): \Gamma \rightarrow \Gamma$ by $u\left(\Gamma^{(r)}\right)=\Gamma^{(r \oplus 1)}$. The controlled queueing system is such that for $i=0,1, \ldots$,

$$
\begin{gathered}
\Gamma_{i+1}=u\left(\Gamma_{i}\right), \quad \kappa_{1, i+1}=\max \left\{0, \kappa_{1, i}+\eta_{1, i}-\xi_{1, i}\right\}, \\
\kappa_{2, i+1}=\max \left\{0, \kappa_{2, i}+\eta_{2, i}+\eta_{3, i}-\xi_{2, i}\right\}, \quad \kappa_{3, i+1}=\kappa_{3, i}+\bar{\xi}_{1, i}-\eta_{3, i}, \\
\bar{\xi}_{1, i+1}=\min \left\{\xi_{1, i}, \kappa_{1, i}+\eta_{1, i}\right\} .
\end{gathered}
$$

The nonlocal description of the input flows as well as that of the saturation flows is given with the help of conditional distributions of the discrete components

$$
\left\{\left(\eta_{1, i}, \eta_{2, i}\right) ; i=0,1, \ldots\right\}, \quad\left\{\left(\xi_{1, i}, \xi_{2, i}\right) ; i=0,1, \ldots\right\}, \quad\left\{\eta_{3, i} ; i=0,1, \ldots\right\}
$$

of the marked point processes

$$
\begin{gathered}
\left\{\left(\tau_{i}, \eta_{1, i}, \eta_{2, i}, \nu_{i}\right) ; i=0,1, \ldots\right\}, \quad\left\{\left(\tau_{i}, \xi_{1, i}, \xi_{2, i}, \nu_{i}\right) ; i=0,1, \ldots\right\}, \\
\left\{\left(\tau_{i}, \eta_{3, i}, \bar{\nu}_{i}\right) ; i=0,1, \ldots\right\},
\end{gathered}
$$

respectively. Here $\nu_{i}=\Gamma_{i}$ and $\bar{\nu}_{i}=\left(\Gamma_{i}, \kappa_{3, i}\right)$ are the marks of customers from the corresponding flows arriving to the system during the interval $\left(\tau_{i}, \tau_{i+1}\right]$.

For $x \geq 0,0 \leq k \leq x$, and $0<\alpha<1$ let

$$
\psi(k ; x, \alpha)=\left(\begin{array}{l}
k \\
x
\end{array}\right) \alpha^{k}(1-\alpha)^{x-k}, \quad \Gamma=\Gamma^{\mathrm{I}} \cup \Gamma^{\mathrm{II}} \cup \Gamma^{\mathrm{III}} \cup \Gamma^{\mathrm{IV}},
$$

where $\Gamma^{\mathrm{I}}, \Gamma^{\mathrm{II}}, \Gamma^{\mathrm{III}}$, and $\Gamma^{\mathrm{IV}}$ are the sets of states of types I, II, III, and IV, respectively. Now we introduce $\varphi_{j}(x, T), T>0, x=0,1, \ldots, j=1,2$, as follows:

$$
\sum_{x=0}^{\infty} z^{x} \varphi_{j}(x, T)=\exp \left\{\lambda_{j} T\left(\sum_{x=1}^{\infty} z^{x} p_{x}^{(j)}-1\right)\right\}, \quad|z|<1 .
$$

Then

$$
\begin{gathered}
\mathrm{P}\left(\left\{\omega: \eta_{j, i}=b\right\} \mid\left\{\omega: \Gamma_{i}=\Gamma^{(r)}\right\}\right)=\varphi_{j}\left(b ; T_{r \oplus 1}\right) \quad \text { if } \quad b=0,1, \ldots, \\
\mathrm{P}\left(\left\{\omega: \xi_{1, i}=0\right\} \mid\left\{\omega: \Gamma_{i}=\Gamma^{(r)}\right\}\right)=1 \quad \text { if } \Gamma^{(r \oplus 1)} \in \Gamma^{\mathrm{I}} \cup \Gamma^{\mathrm{III}}, \\
\mathrm{P}\left(\left\{\omega: \xi_{1, i}=\ell_{r \oplus 1,1}\right\} \mid\left\{\omega: \Gamma_{i}=\Gamma^{(r)}\right\}\right)=1 \quad \text { if } \Gamma^{(r \oplus 1)} \in \Gamma^{\mathrm{II}} \cup \Gamma^{\mathrm{IV}}, \\
\mathrm{P}\left(\left\{\omega: \xi_{2, i}=0\right\} \mid\left\{\omega: \Gamma_{i}=\Gamma^{(r)}\right\}\right)=1 \quad \text { if } \Gamma^{(r \oplus 1)} \in \Gamma^{\mathrm{I}} \cup \Gamma^{\mathrm{II}}, \\
\mathrm{P}\left(\left\{\omega: \xi_{2, i}=\ell_{r \oplus 1,2}\right\} \mid\left\{\omega: \Gamma_{i}=\Gamma^{(r)}\right\}\right)=1 \quad \text { if } \Gamma^{(r \oplus 1)} \in \Gamma^{\mathrm{III}} \cup \Gamma^{\mathrm{IV}}, \\
\mathrm{P}\left(\left\{\omega: \eta_{3, i}=b\right\} \mid\left\{\omega: \Gamma_{i}=\Gamma^{(r)}, \kappa_{3, i}=x\right\}\right)=\psi\left(b ; x, p_{r \oplus 1}\right) \quad \text { if } 0 \leq b \leq x .
\end{gathered}
$$

For nonnegative integers $i, x_{1}, x_{2}, x_{3}, b_{1}, b_{2}, b_{3}, y_{1}, y_{2}$ and $r=1,2, \ldots, n$, we introduce the random events

$$
\begin{aligned}
A_{i}\left(r, x_{1}, x_{2}, x_{3}\right) & =\left\{\omega: \Gamma_{i}=\Gamma^{(r)}, \kappa_{1, i}=x_{1}, \kappa_{2, i}=x_{2}, \kappa_{3, i}=x_{3}\right\}, \\
B_{i}\left(b_{1}, b_{2}, b_{3}, y_{1}, y_{2}\right) & =\left\{\omega: \eta_{1, i}=b_{1}, \eta_{2, i}=b_{2}, \eta_{3, i}=b_{3}, \xi_{1, i}=y_{1}, \xi_{2, i}=y_{2}\right\} .
\end{aligned}
$$


Finally let

$$
\begin{aligned}
\mathrm{P}\left(B_{i}\left(b_{1}, b_{2}, b_{3}, y_{1}, y_{2}\right) \mid \bigcap_{\bar{\iota}=0}^{i} A_{\bar{\iota}}\left(r_{\bar{\iota}}, x_{1, \bar{\iota}}, x_{2, \bar{\iota}}, x_{3, \bar{\iota}}\right)\right) \\
=\mathrm{P}\left(\left\{\omega: \eta_{1, i}=b_{1}\right\} \mid\left\{\omega: \Gamma_{i}=\Gamma^{\left(r_{i}\right)}\right\}\right) \mathrm{P}\left(\left\{\omega: \eta_{2, i}=b_{2}\right\} \mid\left\{\omega: \Gamma_{i}=\Gamma^{\left(r_{i}\right)}\right\}\right) \\
\quad \times \mathrm{P}\left(\left\{\omega: \eta_{3, i}=b_{3}\right\} \mid\left\{\omega: \Gamma_{i}=\Gamma^{\left(r_{i}\right)}, \kappa_{3, i}=x_{i}\right\}\right) \\
\quad \times \mathrm{P}\left(\left\{\omega: \xi_{1, i}=y_{1}\right\} \mid\left\{\omega: \Gamma_{i}=\Gamma^{\left(r_{i}\right)}\right\}\right) \mathrm{P}\left(\left\{\omega: \xi_{2, i}=y_{2}\right\} \mid\left\{\omega: \Gamma_{i}=\Gamma^{\left(r_{i}\right)}\right\}\right)
\end{aligned}
$$

for all nonnegative integers

$i, b_{1}, b_{2}, b_{3}, y_{1}, y_{2}, x_{1,0}, x_{2,0}, x_{3,0}, x_{1,1}, x_{2,1}, x_{3,1}, \ldots, x_{1, i}, x_{2, i}, x_{3, i}$

and arbitrary states $\Gamma^{\left(r_{0}\right)} \in \Gamma, \Gamma^{\left(r_{1}\right)} \in \Gamma, \ldots, \Gamma^{\left(r_{i}\right)} \in \Gamma$.

Theorem 1. Given the probability distribution of the random vector

$$
\left(\Gamma_{0}, \kappa_{1,0}, \kappa_{2,0}, \kappa_{3,0}\right)
$$

the sequence

$$
\left\{\left(\Gamma_{i}, \kappa_{1, i}, \kappa_{2, i}, \kappa_{3, i}\right) ; i=0,1, \ldots\right\}
$$

is a Markov chain.

Proof. The law of total probability and assumption (1) imply that

$$
\begin{aligned}
\mathrm{P}\left(A_{i+1}\right. & \left.\left(r_{i} \oplus 1, x_{1, i+1}, x_{2, i+1}, x_{3, i+1}\right) \mid W(i)\right) \\
= & \sum_{b_{1}=0}^{\infty} \sum_{b_{2}=0}^{\infty} \sum_{b_{3}=0}^{\infty} \sum_{y_{1}=0}^{\infty} \sum_{y_{2}=0}^{\infty} \mathrm{P}\left(B_{i}\left(b_{1}, b_{2}, b_{3}, y_{1}, y_{2}\right) \mid W(i)\right) \\
& \times \mathrm{P}\left(A_{i+1}\left(r_{i} \oplus 1, x_{1, i+1}, x_{2, i+1}, x_{3, i+1}\right) \mid W(i) \cap B_{i}\left(b_{1}, b_{2}, b_{3}, y_{1}, y_{2}\right)\right) \\
= & \sum_{b_{1}=0}^{\infty} \sum_{b_{2}=0}^{\infty} \sum_{b_{3}=0}^{\infty} \sum_{y_{1}=0}^{\infty} \sum_{y_{2}=0}^{\infty} \mathrm{P}\left(\left\{\omega: \eta_{1, i}=b_{1}\right\} \mid\left\{\omega: \Gamma_{i}=\Gamma^{\left(r_{i}\right)}\right\}\right) \\
& \times \mathrm{P}\left(\left\{\omega: \eta_{2, i}=b_{2}\right\} \mid\left\{\omega: \Gamma_{i}=\Gamma^{\left(r_{i}\right)}\right\}\right) \\
& \times \mathrm{P}\left(\left\{\omega: \eta_{3, i}=b_{3}\right\} \mid\left\{\omega: \Gamma_{i}=\Gamma^{\left(r_{i}\right)}, \kappa_{3, i}=x_{i}\right\}\right) \\
& \times \mathrm{P}\left(\left\{\omega: \xi_{1, i}=y_{1}\right\} \mid\left\{\omega: \Gamma_{i}=\Gamma^{\left(r_{i}\right)}\right\}\right) \mathrm{P}\left(\left\{\omega: \xi_{2, i}=y_{2}\right\} \mid\left\{\omega: \Gamma_{i}=\Gamma^{\left(r_{i}\right)}\right\}\right) \\
& \times \mathrm{P}\left(\left\{\omega: x_{1, i+1}=\max \left\{0, x_{1, i}+b_{1}-y_{1}\right\}, x_{2, i+1}=\max \left\{0, x_{2, i}+b_{2}+b_{3}-y_{2}\right\},\right.\right. \\
&
\end{aligned}
$$

for all $\Gamma^{\left(r_{0}\right)} \in \Gamma, \Gamma^{\left(r_{1}\right)} \in \Gamma, \ldots, \Gamma^{\left(r_{i}\right)} \in \Gamma$ and all nonnegative numbers

$$
x_{1,0}, x_{2,0}, x_{3,0}, x_{1,1}, x_{2,1}, x_{3,1}, \ldots, x_{1, i+1}, x_{2, i+1}, x_{3, i+1},
$$

where

$$
W(i)=\bigcap_{\bar{\iota}=0}^{i} A_{\bar{\iota}}\left(r_{\bar{\iota}}, x_{1, \bar{\iota}}, x_{2, \bar{\iota}}, x_{3, \bar{\iota}}\right) .
$$


Similarly

$$
\begin{aligned}
& \mathrm{P}\left(A_{i+1}\right.\left.\left(r_{i} \oplus 1, x_{1, i+1}, x_{2, i+1}, x_{3, i+1}\right) \mid A_{i}\left(r_{i}, x_{1, i}, x_{2, i}, x_{3, i}\right)\right) \\
&= \sum_{b_{1}=0}^{\infty} \sum_{b_{2}=0}^{\infty} \sum_{b_{3}=0}^{\infty} \sum_{y_{1}=0}^{\infty} \sum_{y_{2}=0}^{\infty} \mathrm{P}\left(\left\{\omega: \eta_{1, i}=b_{1}\right\} \mid\left\{\omega: \Gamma_{i}=\Gamma^{\left(r_{i}\right)}\right\}\right) \\
& \times \mathrm{P}\left(\left\{\omega: \eta_{2, i}=b_{2}\right\} \mid\left\{\omega: \Gamma_{i}=\Gamma^{\left(r_{i}\right)}\right\}\right) \\
& \times \mathrm{P}\left(\left\{\omega: \eta_{3, i}=b_{3}\right\} \mid\left\{\omega: \Gamma_{i}=\Gamma^{\left(r_{i}\right)}, \kappa_{3, i}=x_{i}\right\}\right) \\
& \times \mathrm{P}\left(\left\{\omega: \xi_{1, i}=y_{1}\right\} \mid\left\{\omega: \Gamma_{i}=\Gamma^{\left(r_{i}\right)}\right\}\right) \mathrm{P}\left(\left\{\omega: \xi_{2, i}=y_{2}\right\} \mid\left\{\omega: \Gamma_{i}=\Gamma^{\left(r_{i}\right)}\right\}\right) \\
& \times \mathrm{P}\left(\left\{\omega: x_{1, i+1}=\max \left\{0, x_{1, i}+b_{1}-y_{1}\right\},\right.\right. \\
& x_{2, i+1}=\max \left\{0, x_{2, i}+b_{2}+b_{3}-y_{2}\right\}, \\
&\left.\left.x_{3, i+1}=x_{3, i}+\min \left\{y_{1}, x_{1, i}+b_{1}\right\}-b_{3}\right\}\right) .
\end{aligned}
$$

The right hand sides of the latter two equations coincide, whence we conclude that the conditional probabilities on the left hand sides coincide, too.

Put $Q_{i}\left(r ; x_{1}, x_{2}, x_{3}\right)=\mathrm{P}\left(A_{i}\left(r, x_{1}, x_{2}, x_{3}\right)\right)$. The symbols $w_{1}, w_{2}, w_{3}, x_{1}, x_{2}, x_{3}, b_{1}$, $b_{2}$, and $c$ denote nonnegative integers. From (3), we obtain

$$
Q_{i+1}\left(r \oplus 1 ; w_{1}, w_{2}, w_{3}\right)=\sum Q_{i}\left(r ; x_{1}, x_{2}, x_{3}\right) \varphi_{1}\left(b_{1} ; T_{r \oplus 1}\right) \varphi_{2}\left(b_{2} ; T_{r \oplus 1}\right) \psi\left(c ; x_{3}, p_{r \oplus 1}\right),
$$

where the summation is taken over all nonnegative integers $x_{1}, x_{2}, x_{3}, b_{1}, b_{2}$, and $c$ such that

$$
\begin{gathered}
w_{1}=\max \left\{0, x_{1}+b_{1}-y_{1}\right\}, w_{2}=\max \left\{0, x_{2}+b_{2}+c-y_{2}\right\}, \\
w_{3}=x_{3}+\min \left\{y_{1}, x_{1}+b_{1}\right\}-c,
\end{gathered}
$$

and $c \leq x_{3}$. The number $y_{j}$ above is equal to 0 if the customers from the queue $O_{j}$ are not served in the state $\Gamma^{(r \oplus 1)}$, while $y_{j}=\ell_{r \oplus 1, j}$ otherwise.

Now let $\Gamma^{(r \oplus 1)} \in \Gamma^{\mathrm{I}}$. Then $y_{1}=y_{2}=0$ and the system of equations (4) reduces to

$$
w_{1}=x_{1}+b_{1}, \quad w_{2}=x_{2}+b_{2}+c, \quad w_{3}=x_{3}-c
$$

for $w_{1} \geq 0, w_{2} \geq 0$, and $w_{3} \geq 0$. The solution of this system is given by

$$
b_{1}=w_{1}-x_{1}, \quad b_{2}=w_{2}-c-x_{2}, \quad x_{3}=w_{3}+c
$$

with $0 \leq x_{1} \leq w_{1}, 0 \leq c \leq w_{2}$, and $0 \leq x_{2} \leq w_{2}-c$. Therefore

$$
\begin{aligned}
& Q_{i+1}(\left.r \oplus 1 ; w_{1}, w_{2}, w_{3}\right) \\
&=\sum_{x_{1}=0}^{w_{1}} \sum_{c=0}^{w_{2}} \sum_{x_{2}=0}^{w_{2}-c} Q_{i}\left(r ; x_{1}, x_{2}, w_{3}+c\right) \\
& \quad \times \varphi_{1}\left(w_{1}-x_{1} ; T_{r \oplus 1}\right) \varphi_{2}\left(w_{2}-c-x_{2} ; T_{r \oplus 1}\right) \psi\left(c ; w_{3}+c, p_{r \oplus 1}\right) .
\end{aligned}
$$

Similar reasoning for $\Gamma^{(r \oplus 1)} \in \Gamma^{\mathrm{II}}$ proves that if $w_{3} \geq 0$,

(6)

$$
\begin{aligned}
& Q_{i+1}\left(r \oplus 1 ; 0,0, w_{3}\right) \\
&=\left.\sum_{x_{1}=0}^{\min \left\{w_{3}, \ell_{r} \oplus 1,1\right.}\right\} \sum_{b_{1}=0}^{\min \left\{w_{3}, \ell_{r \oplus 1,1}\right\}-x_{1}} Q_{i}\left(r ; x_{1}, 0, w_{3}-x_{1}-b_{1}\right) \\
& \times \varphi_{1}\left(b_{1} ; T_{r \oplus 1}\right) \varphi_{2}\left(0 ; T_{r \oplus 1}\right) \psi\left(0 ; w_{3}-x_{1}-b_{1}, p_{r \oplus 1}\right) ;
\end{aligned}
$$


if $w_{2} \geq 1$,

$$
Q_{i+1}\left(r \oplus 1 ; 0, w_{2}, 0\right)
$$

$$
=\sum_{c=0}^{w_{2}} \sum_{x_{2}=0}^{w_{2}-c} \varphi_{1}\left(0 ; T_{r \oplus 1}\right) \varphi_{2}\left(w_{2}-x_{2}-c ; T_{r \oplus 1}\right) \psi\left(c ; c, p_{r \oplus 1}\right) Q_{i}\left(r ; 0, x_{2}, c\right) ;
$$

if $w_{2} \geq 1$ and $w_{3} \geq 1$,

$$
\begin{aligned}
& Q_{i+1}\left(r \oplus 1 ; 0, w_{2}, w_{3}\right) \\
& =\sum_{c=0}^{w_{2}} \sum_{x_{2}=0}^{w_{2}-c} \sum_{x_{1}=0}^{\min \left\{w_{3}+c, \ell_{r \oplus 1,1}\right\}} \sum_{b_{1}=0}^{\min \left\{w_{3}+c, \ell_{r \oplus 1,1}\right\}-x_{1}} \varphi_{1}\left(b_{1} ; T_{r \oplus 1}\right) \\
& \times Q_{i}\left(r ; x_{1}, x_{2}, w_{3}+c-x_{1}-b_{1}\right) \varphi_{2}\left(w_{2}-c-x_{2} ; T_{r \oplus 1}\right) \\
& \times \psi\left(c ; w_{3}+c-x_{1}-b_{1}, p_{r \oplus 1}\right) ;
\end{aligned}
$$

and if $w_{1} \geq 1, w_{2} \geq 0$, and $w_{3} \geq \ell_{r \oplus 1,1}$,

$$
\begin{aligned}
Q_{i+1}\left(r \oplus 1 ; w_{1}, w_{2}, w_{3}\right) \\
=\sum_{x_{1}=0}^{w_{1}+\ell_{r \oplus 1,1}} \sum_{c=0}^{w_{2}} \sum_{x_{2}=0}^{w_{2}-c} Q_{i}\left(r ; x_{1}, x_{2}, c+w_{3}-\ell_{r \oplus, 1}\right) \\
\times \varphi_{1}\left(w_{1}+\ell_{r \oplus 1,1}-x_{1} ; T_{r \oplus 1}\right) \varphi_{2}\left(w_{2}-c-x_{2} ; T_{r \oplus 1}\right) \\
\times \psi\left(c ; c+w_{3}-\ell_{r \oplus 1,1}, p_{r \oplus 1}\right)
\end{aligned}
$$

In the case $\Gamma^{(r \oplus 1)} \in \Gamma^{\mathrm{III}}$,

$$
\begin{aligned}
& Q_{i+1}(\left(r \oplus 1 ; w_{1}, 0, w_{3}\right) \\
&=\sum_{x_{1}}^{w_{1}} \sum_{x_{2}=0}^{\ell_{r \oplus 1,2}} \sum_{c=0}^{\ell_{r \oplus 1,2}-x_{2}} \sum_{\substack{\ell_{2}=0 \\
\times Q_{i}, 2}}^{\times x_{2}-c} \varphi_{1}\left(w_{1}-x_{1}, x_{2}, w_{3}+c\right) T_{r}\left(b_{2} ; T_{r \oplus 1}\right) \psi\left(c ; w_{3}+c, p_{r \oplus 1}\right)
\end{aligned}
$$

if $w_{1} \geq 0$ and $w_{3} \geq 0$, and

$$
\begin{aligned}
& Q_{i+1}\left(r \oplus 1 ; w_{1}, w_{2}, w_{3}\right)=\sum_{x_{1}=0}^{w_{1}} \sum_{c=0}^{w_{2}+\ell_{r \oplus 1,2}} \sum_{x_{2}=0}^{w_{2}+\ell_{r \oplus 1,2}-c} Q_{i}\left(r ; x_{1}, x_{2}, w_{3}+c\right) \varphi_{1}\left(w_{1}-x_{1} ; T_{r \oplus 1}\right) \\
& \times \varphi_{2}\left(w_{2}+\ell_{r \oplus 1,2}-x_{2}-c ; T_{r \oplus 1}\right) \psi\left(c ; w_{3}+x, p_{r \oplus 1}\right)
\end{aligned}
$$

if $w_{1} \geq 0, w_{2} \geq 1$, and $w_{3} \geq 0$.

In the case $\Gamma^{(r \oplus 1)} \in \Gamma^{\mathrm{IV}}$, for $w_{3} \geq 0$,

$$
\begin{aligned}
Q_{i+1} & \left(r \oplus 1 ; 0,0, w_{3}\right) \\
= & \sum_{x_{1}=0}^{\min \left\{w_{3}, \ell_{r \oplus 1,1}\right\}} \sum_{b_{1}=0}^{\min \left\{w_{3}, \ell_{r \oplus 1,1}\right\}} \sum_{c=0} \sum_{Q_{i}\left(r ; x_{1}, x_{2}, w_{3}+c-x_{1}-b_{1}\right)}^{\ell_{r \oplus 1,2}} \sum_{\varphi_{2}\left(b_{2} ; T_{r \oplus 1}\right)}^{\ell_{r \oplus 1,2}-c \ell_{r \oplus 1,2}-c-x_{2}} \sum_{b_{2}=0} \varphi_{1}\left(b_{1} ; T_{r \oplus 1}\right)
\end{aligned}
$$


for $w_{2} \geq 1$ and $w_{3} \geq 0$,

$$
\begin{aligned}
Q_{i+1}\left(\Gamma^{(r \oplus 1)} ; 0, w_{2}, w_{3}\right) \\
=\sum_{x_{1}=0}^{\min \left\{\ell_{r \oplus 1,1}, w_{3}\right\}} \sum_{b_{1}=0}^{\min \left\{\ell_{r \oplus 1,1}, w_{3}\right\}-x_{1}} \sum_{c=0}^{w_{2}+\ell_{r \oplus 1,2}} \sum_{x_{2}=0}^{\ell_{r \oplus 1,2}-c} \varphi_{1}\left(b_{1} ; T_{r \oplus 1}\right) \\
\quad \times \psi\left(c ; w_{3}+c-x_{1}-x_{2}, w_{3}+c-x_{1}-b_{1}\right) \varphi_{2}\left(w_{2}+\ell_{r \oplus 1,2}-x_{2}-c ; T_{r \oplus 1}\right)
\end{aligned}
$$

for $w_{1} \geq 1$ and $w_{3} \geq \ell_{r \oplus 1,1}$,

$$
\begin{gathered}
Q_{i+1}\left(\Gamma^{(r \oplus 1)} ; w_{1}, 0, w_{3}\right) \\
=\sum_{x_{1}=0}^{w_{1}+\ell_{r \oplus 1,1}} \sum_{c=0}^{\ell_{r \oplus 1,2}} \sum_{\substack{\ell_{2}=0 \\
r \oplus 1,2}} \sum_{b_{2}=0} \sum_{\varphi_{1}\left(w_{1}+\ell_{r \oplus 1,1}-x_{1} ; T_{r \oplus 1}\right) \psi\left(c ; w_{3}+c-\ell_{r \oplus 1,1}, p_{r \oplus 1}\right)} Q_{i}\left(\Gamma^{(r)} ; x_{1}, x_{2}, w_{3}+c-\ell_{r \oplus 1,1}\right) \\
\times \varphi_{2}\left(b_{2} ; T_{r \oplus 1}\right) ;
\end{gathered}
$$

and for $w_{1} \geq 1, w_{2} \geq 1$, and $w_{3} \geq \ell_{r \oplus 1,1}$,

$$
\begin{aligned}
& Q_{i+1}\left(\Gamma^{(r \oplus 1)} ; w_{1}, w_{2}, w_{3}\right) \\
& =\sum_{x_{1}=0}^{w_{1}+\ell_{r \oplus 1,1}} \sum_{c=0}^{\ell_{r \oplus 1,2}} \sum_{x_{2}=0}^{\ell_{r \oplus 1,2}-c} Q_{i}\left(\Gamma^{(r)} ; x_{1}, x_{2}, w_{3}+c-\ell_{r \oplus 1,1}\right) \\
& \times \varphi_{1}\left(w_{1}+\ell_{r \oplus 1,1}-x_{1} ; T_{r \oplus 1}\right) \varphi_{2}\left(w_{2}+\ell_{r \oplus 1,2}-x_{2}-c ; T_{r \oplus 1}\right) \\
& \times \psi\left(c ; w_{3}+c-\ell_{r \oplus 1,1}, p_{r \oplus 1}\right) \text {. }
\end{aligned}
$$

Finally,

$$
Q_{i+1}\left(r \oplus 1 ; w_{1}, w_{2}, w_{3}\right)=0
$$

if $\Gamma^{(r \oplus 1)} \in \Gamma^{\mathrm{II}} \cup \Gamma^{\mathrm{IV}}$ and $w_{1} \geq 1, w_{2} \geq 0,0 \leq w_{3} \leq \ell_{r \oplus 1,1}-1$. Thus we have proved the theorem for the marginal probability distributions of the Markov chain (2).

Theorem 2. The probabilities $Q_{i}\left(r ; x_{1}, x_{2}, x_{3}\right)$ satisfy recurrence equations (5) -(16) with respect to $i=0,1, \ldots$.

An arbitrary element of the set $X$ is denoted by $w=\left(w_{1}, w_{2}, w_{3}\right)$. Let

$$
\begin{aligned}
& E_{1}^{\prime}=\left\{\left(\Gamma^{(r)}, w\right): \Gamma^{(r)} \in \Gamma^{\mathrm{II}} \cup \Gamma^{\mathrm{IV}}, w_{1}>0, w_{3}<\ell_{r, 1}\right\}, \\
& E_{1}=\left\{(\gamma, w): \gamma \in \Gamma^{\mathrm{I}} \cup \Gamma^{\mathrm{III}}, w \in X\right\}, \\
& E_{2}=\left\{(\gamma, w): \gamma \in \Gamma^{\mathrm{II}} \cup \Gamma^{\mathrm{IV}}, w_{1}=0\right\}, \\
& E_{3}=\left\{\left(\Gamma^{(r)}, w\right): \Gamma^{(r)} \in \Gamma^{\mathrm{II}} \cup \Gamma^{\mathrm{IV}}, w_{1}>0, w_{3} \geq \ell_{r, 1}\right\} .
\end{aligned}
$$

Corollary 1. The set of states of the Markov chain (2) is the union of the nonclosed set $E_{1}^{\prime}$ of inessential states and the closed set $E_{1} \cup E_{2} \cup E_{3}$ of essential periodic states with period $n$.

Consider the probability generating functions

$$
\begin{gathered}
\Psi_{i}\left(r ; z_{1}, z_{2}, z_{3}\right)=\sum_{x_{1}=0}^{\infty} \sum_{x_{2}=0}^{\infty} \sum_{x_{3}=0}^{\infty} z_{1}^{x_{1}} z_{2}^{x_{2}} z_{3}^{x_{3}} Q_{i}\left(r ; x_{1}, x_{2}, x_{3}\right), \\
q_{r, j}\left(z_{j}\right)=\exp \left\{\lambda_{j} T_{r}\left(\sum_{x=1}^{\infty} p_{x}^{(j)} z_{j}^{x}-1\right)\right\}
\end{gathered}
$$

for $r=1,2, \ldots, n, j=1,2$, and $\left|z_{1}\right| \leq 1,\left|z_{2}\right| \leq 1,\left|z_{3}\right| \leq 1$. 
Theorem 3. The following recurrence relations for the probability generating functions hold with respect to $i=0,1, \ldots$ :

$$
\begin{aligned}
& \Psi_{i+1}\left(r \oplus 1 ; z_{1}, z_{2}, z_{3}\right)=q_{r \oplus 1,1}\left(z_{1}\right) q_{r \oplus 1,2}\left(z_{2}\right) \Psi_{i}\left(r ; z_{1}, z_{2}, z_{2} p_{r \oplus 1}+z_{3}\left(1-p_{r \oplus 1}\right)\right) \\
& \text { if } \Gamma^{(r \oplus 1)} \in \Gamma^{\mathrm{I}} ; \\
& \Psi_{i+1}\left(r \oplus 1 ; z_{1}, z_{2}, z_{3}\right) \\
& =\left(\frac{z_{3}}{z_{1}}\right)^{\ell_{r \oplus 1,1}} q_{r \oplus 1,1}\left(z_{1}\right) q_{r \oplus 1,2}\left(z_{2}\right) \Psi_{i}\left(r ; z_{1}, z_{2}, z_{2} p_{r \oplus 1}+z_{3}\left(1-p_{r \oplus 1}\right)\right) \\
& \quad+q_{r \oplus 1,2}\left(z_{2}\right) \sum_{x_{1}=0}^{\ell_{r \oplus 1,1}} \sum_{x_{2}=0}^{\infty} \sum_{x_{3}=0}^{\infty} Q_{i}\left(r ; x_{1}, x_{2}, x_{3}\right) z_{2}^{x_{2}}\left(z_{2} p_{r \oplus 1}+z_{3}\left(1-p_{r \oplus 1}\right)\right)^{x_{3}} \\
& \times \sum_{\ell_{r \oplus 1,1}-x_{1}}^{\ell_{1}=0}\left(z_{3}^{x_{1}+b_{1}}-z_{1}^{x_{1}+b_{1}-\ell_{r \oplus 1,1}} z_{3}^{\ell_{r \oplus 1,1}}\right) \varphi_{1}\left(b_{1} ; T_{r \oplus 1}\right)
\end{aligned}
$$

if $\Gamma^{(r \oplus 1)} \in \Gamma^{\mathrm{II}}$

$$
\begin{aligned}
\Psi_{i+1}\left(r \oplus 1 ; z_{1}, z_{2}, z_{3}\right) & \\
= & z_{2}^{-\ell_{r \oplus 1,2}} q_{r \oplus 1,1}\left(z_{1}\right) q_{r \oplus 1,2}\left(z_{2}\right) \Psi_{i}\left(r ; z_{1}, z_{2}, z_{2} p_{r \oplus 1}+z_{3}\left(1-p_{r \oplus 1}\right)\right) \\
& +q_{r \oplus 1,1}\left(z_{1}\right) \sum_{x_{1}=0}^{\infty} \sum_{x_{2}=0}^{\ell_{r \oplus 1,2}} \sum_{x_{3}=0}^{\infty} Q_{i}\left(r ; x_{1}, x_{2}, x_{3}\right) \\
& \times \sum_{c=0}^{\min \left\{x_{3}, \ell_{r \oplus 1,2}-x_{2}\right\}} \sum_{\ell_{r \oplus 1,2}-x_{2}-c} \sum_{b_{2}=0} r\left(x_{1}, x_{2}, x_{3}, c, b_{2}\right)
\end{aligned}
$$

if $\Gamma^{(r \oplus 1)} \in \Gamma^{\mathrm{III}}$, where

$$
r\left(x_{1}, x_{2}, x_{3}, c, b_{2}\right)=z_{1}^{x_{1}}\left(1-z_{2}^{x_{2}+b_{2}+c-\ell_{r \oplus 1,2}}\right) z_{3}^{x_{3}-c} \varphi_{2}\left(b_{2} ; T_{r \oplus 1}\right) \psi\left(c ; x_{3}, p_{r \oplus 1}\right)
$$

and

$$
\begin{aligned}
\Psi_{i+1}( & \left.\oplus 1 ; z_{1}, z_{2}, z_{3}\right) \\
= & \left(\frac{z_{3}}{z_{1}}\right)^{\ell_{r \oplus 1,1}} z_{2}^{-\ell_{r \oplus 1,2}} q_{r \oplus 1,1}\left(z_{1}\right) q_{r \oplus 1,2}\left(z_{2}\right) \Psi_{i}\left(r ; z_{1}, z_{2}, z_{2} p_{r \oplus 1}+z_{3}\left(1-p_{r \oplus 1}\right)\right) \\
& +\sum_{x_{1}=0}^{\ell_{r \oplus 1,1}} \sum_{x_{2}=0}^{\infty} \sum_{x_{3}=0}^{\infty} Q_{i}\left(r ; x_{1}, x_{2}, x_{3}\right) g_{1}\left(x_{1}, x_{2}, x_{3}, r\right) \\
& +\sum_{x_{1}=0}^{\infty} \sum_{x_{2}=0}^{\ell_{r \oplus 1,2}} \sum_{x_{3}=0}^{\infty} Q_{i}\left(r ; x_{1}, x_{2}, x_{3}\right) g_{2}\left(x_{1}, x_{2}, x_{3}, r\right) \\
& +\sum_{\ell_{1}=0}^{\ell_{1,1}} \sum_{\ell_{r}=0}^{\ell_{r}=1,2} \sum_{x_{3}=0}^{\infty} Q_{i}\left(r ; x_{1}, x_{2}, x_{3}\right) g_{3}\left(x_{1}, x_{2}, x_{3}, r\right)
\end{aligned}
$$


if $\Gamma^{(r \oplus 1)} \in \Gamma^{\mathrm{IV}}$, where

$$
\begin{aligned}
& g_{1}\left(x_{1}, x_{2}, x_{3}, r\right)=\sum_{b_{1}=0}^{\ell_{r \oplus 1,1}-x_{1}} \sum_{c=m_{1}}^{x_{3}} \sum_{b_{2}=m_{2}}^{\infty} z_{2}^{x_{2}+b_{2}+c-\ell_{r \oplus 1,2}} \\
& \times\left(z_{3}^{x_{1}+x_{3}+b_{1}-c}-z_{1}^{x_{1}+b_{1}-\ell_{r \oplus 1,1}} z_{3}^{x_{3}+\ell_{r \oplus 1,1}-c}\right) \\
& \times \varphi_{1}\left(b_{1} ; T_{r \oplus 1,1}\right) \varphi_{2}\left(b_{2} ; T_{r \oplus 1}\right) \psi\left(c ; x_{3}, p_{r \oplus 1}\right), \\
& m_{1}=\max \left\{0, \ell_{r \oplus 1,2}-x_{2}\right\}, \quad m_{2}=\max \left\{0, l\left(r, x_{2}\right)\right\}, \quad l\left(r, x_{2}\right)=\ell_{r \oplus 1,2}-x_{2}-c, \\
& g_{2}\left(x_{1}, x_{2}, x_{3}, r\right)=\sum_{b_{1}=m_{3}}^{\infty} \sum_{c=0}^{m_{4}} \sum_{b_{2}=0}^{l\left(r, x_{2}\right)} \varphi_{1}\left(b_{1} ; T_{r \oplus 1}\right) \varphi_{2}\left(b_{2} ; T_{r \oplus 1}\right) \psi\left(c ; x_{3}, p_{r \oplus 1}\right) z_{1}^{x_{1}+b_{1}-\ell_{r \oplus 1,1}} \\
& \times\left(1-z_{2}^{x_{2}+b_{2}+c-\ell_{r \oplus 1,2}}\right) z_{3}^{x_{3}+\ell_{r \oplus 1,1}-c}, \\
& m_{3}=\max \left\{0, \ell_{r \oplus 1,1}-x_{1}\right\}, \quad m_{4}=\min \left\{x_{3}, \ell_{r \oplus 1,2}-x_{2}\right\}, \\
& g_{3}\left(x_{1}, x_{2}, x_{3}, r\right)=\sum_{b_{1}=0}^{\ell_{r \oplus 1,1}-x_{1}} \sum_{c=0}^{m_{5}} \sum_{b_{2}=0}^{l\left(r, x_{2}\right)} \varphi_{1}\left(b_{1} ; T_{r \oplus 1}\right) \varphi_{2}\left(b_{2} ; T_{r \oplus 1}\right) \psi\left(c ; x_{3}, p_{r \oplus 1}\right) \\
& \times\left(z_{3}^{x_{1}+x_{3}+b_{1}-c}-z_{1}^{x_{1}+b_{1}-\ell_{r \oplus 1,1}} z_{2}^{x_{2}+b_{2}+c-\ell_{r \oplus 1,2}} z_{3}^{x_{3}+\ell_{r \oplus 1,1}-c}\right), \\
& m_{5}=\min \left\{x_{3}, \ell_{r \oplus 1,2}-x_{2}\right\} .
\end{aligned}
$$

Proof. Denote by $\mathrm{E}(\cdot)$ the operator of the mathematical expectation. Let $I_{B}(\omega)$ be the indicator of an event $B \in \mathfrak{F}$ and let $\mathrm{E}(\theta ; B)=\mathrm{E}\left(\theta I_{B}\right)$ for a random variable $\theta$. Then the probability generating functions $\Psi_{i}\left(r ; z_{1}, z_{2}, z_{3}\right), r=1,2, \ldots, n$, can be rewritten as follows:

$$
\Psi_{i}\left(r ; z_{1}, z_{2}, z_{3}\right)=\mathrm{E}\left(z_{1}^{\kappa_{1, i}} z_{2}^{\kappa_{2, i}} z_{3}^{\kappa_{3, i}} ;\left\{\omega: \Gamma_{i}=\Gamma^{(r)}\right\}\right)
$$

Let $x=\left(x_{1}, x_{2}, x_{3}\right)$ be an arbitrary element of the set $X$. We exhibit the general proof for the most complicated case, namely for $\Gamma^{(r \oplus 1)} \in \Gamma^{\mathrm{IV}}$. The other cases can be treated in an analogous way.

If $\Gamma^{(r \oplus 1)} \in \Gamma^{\mathrm{IV}}$, then

$$
\begin{aligned}
\Psi_{i+1}( & \left(r 1 ; z_{1}, z_{2}, z_{3}\right)=\sum_{x \in X} Q_{i}\left(r ; x_{1}, x_{2}, x_{3}\right) \mathrm{E}\left(z_{1}^{m_{6}} z_{2}^{m_{7}} z_{3}^{x_{3}+m_{8}-\eta_{3, i}} \mid A_{i}\left(r ; x_{1}, x_{2}, x_{3}\right)\right) \\
= & \sum_{x \in X} \mathrm{E}\left(Z\left(x_{1}, x_{2}, x_{3}\right) \mid A_{i}\left(r ; x_{1}, x_{2}, x_{3}\right)\right) Q_{i}\left(r ; x_{1}, x_{2}, x_{3}\right) \\
& +\sum_{x \in X} \mathrm{E}\left(z_{1}^{m_{6}} z_{2}^{m_{7}} z_{3}^{x_{3}+m_{8}-\eta_{3, i}}-Z\left(x_{1}, x_{2}, x_{3}\right) \mid A_{i}\left(r ; x_{1}, x_{2}, x_{3}\right)\right) Q_{i}\left(r ; x_{1}, x_{2}, x_{3}\right),
\end{aligned}
$$

where

$$
\begin{gathered}
m_{6}=\max \left\{0, x_{1}+\eta_{1, i}-\ell_{r \oplus 1,1}\right\}, \quad m_{7}=\max \left\{0, x_{2}+\eta_{2, i}+\eta_{3, i}-\ell_{r \oplus 1,2}\right\}, \\
m_{8}=\min \left\{x_{1}+\eta_{1, i}, \ell_{r \oplus 1,1}\right\}, \\
Z\left(x_{1}, x_{2}, x_{3}\right)=z_{1}^{x_{1}+\eta_{1, i}-\ell_{r \oplus 1,1}} z_{2}^{x_{2}+\eta_{2, i}+\eta_{3, i}-\ell_{r \oplus 1,2}} z_{3}^{x_{3}+\ell_{r \oplus 1,1}-\eta_{3, i}} .
\end{gathered}
$$

The first term on the right hand side is equal to

$$
\left(\frac{z_{3}}{z_{1}}\right)^{\ell_{r \oplus 1,1}} z_{2}^{-\ell_{r \oplus 1,2}} q_{r \oplus 1,1}\left(z_{1}\right) q_{r \oplus 1,2}\left(z_{2}\right) \Psi_{i}\left(r ; z_{1}, z_{2}, z_{2} p_{r \oplus 1}+z_{3}\left(1-p_{r \oplus 1}\right)\right) .
$$


Since

$$
\begin{aligned}
& I_{\left\{\eta_{1, i} \leq \ell_{r \oplus 1,1}-x_{1}, \eta_{2, i}+\eta_{3, i}>\ell_{r \oplus 1,2}-x_{2}\right\}}+I_{\left\{\eta_{1, i}>\ell_{r \oplus 1,1}-x_{1}, \eta_{2, i}+\eta_{3, i} \leq \ell_{r \oplus 1,2}-x_{2}\right\}} \\
& \quad+I_{\left\{\eta_{1, i} \leq \ell_{r \oplus 1,1}-x_{1}, \eta_{2, i}+\eta_{3, i} \leq \ell_{r \oplus 1,2}-x_{2}\right\}}+I_{\left\{\eta_{1, i}>\ell_{r \oplus 1,1}-x_{1}, \eta_{2, i}+\eta_{3, i}>\ell_{r \oplus 1,2}-x_{2}\right\}}=1
\end{aligned}
$$

and

$$
z_{1}^{m_{6}} z_{2}^{m_{7}} z_{3}^{x_{3}+m_{8}-\eta_{3, i}}=z_{1}^{x_{1}+\eta_{1, i}-\ell_{r \oplus 1,1}} z_{2}^{x_{2}+\eta_{2, i}+\eta_{3, i}-\ell_{r \oplus 1,2}} z_{3}^{x_{3}+\ell_{r \oplus 1,1}-\eta_{3, i}}
$$

for $\eta_{1, i}>\ell_{r \oplus 1,1}-x_{1}$ and $\eta_{2, i}+\eta_{3, i}>\ell_{r \oplus 1,2}-x_{2}$, we obtain the following equation:

$$
\begin{aligned}
& \mathrm{E}\left(z_{1}^{m_{6}} z_{2}^{m_{7}} z_{3}^{x_{3}+m_{8}-\eta_{3, i}}-Z\left(x_{1}, x_{2}, x_{3}\right)\right) \\
&=\mathrm{E}\left(\left(z_{2}^{x_{2}+\eta_{2, i}+\eta_{3, i}-\ell_{r \oplus 1,2}} z_{3}^{x_{3}+x_{1}+\eta_{1, i}-\eta_{3, i}}-Z\left(x_{1}, x_{2}, x_{3}\right)\right)\right. \\
&\left.\quad \times I_{\left\{\eta_{1, i} \leq \ell_{r \oplus 1,1}-x_{1}, \eta_{2, i}+\eta_{3, i}>\ell_{r \oplus 1,2}-x_{2}\right\}} \mid A_{i}\left(r ; x_{1}, x_{2}, x_{3}\right)\right) \\
&+\mathrm{E}\left(\left(z_{1}^{x_{1}+\eta_{1, i}-\ell_{r \oplus 1,1}} z_{3}^{x_{3}+\ell_{r \oplus 1,1}-\eta_{3, i}}-Z\left(x_{1}, x_{2}, x_{3}\right)\right)\right. \\
&\left.\quad \times I_{\left\{\eta_{1, i}>\ell_{r \oplus 1,1}-x_{1}, \eta_{2, i}+\eta_{3, i} \leq \ell_{r \oplus 1,2}-x_{2}\right\}} \mid A_{i}\left(r ; x_{1}, x_{2}, x_{3}\right)\right) \\
&+\mathrm{E}\left(\left(z_{3}^{x_{3}+x_{1}+\eta_{1, i}-\eta_{3, i}}-Z\left(x_{1}, x_{2}, x_{3}\right)\right)\right. \\
&\left.\quad \times I_{\left\{\eta_{1, i} \leq \ell_{r \oplus 1,1}-x_{1}, \eta_{2, i}+\eta_{3, i} \leq \ell_{r \oplus 1,2}-x_{2}\right\}} \mid A_{i}\left(r ; x_{1}, x_{2}, x_{3}\right)\right) .
\end{aligned}
$$

Note that some terms above are vanishing for some combinations of values of $x_{1}, x_{2}$, and $x_{3}$. For example, the event $\eta_{1, i} \leq \ell_{r \oplus 1,1}-x_{1}$ is impossible for $x_{1}>\ell_{r \oplus 1,1}$. This implies that

$$
\begin{aligned}
\sum_{x \in X} Q_{i}\left(r ; x_{1}, x_{2}, x_{3}\right) \mathrm{E}\left(\left(z_{2}^{x_{2}+\eta_{2, i}+\eta_{3, i}-\ell_{r \oplus 1,2}} z_{3}^{x_{3}+x_{1}+\eta_{1, i}-\eta_{3, i}}-Z\left(x_{1}, x_{2}, x_{3}\right)\right)\right. \\
\left.\quad \times I_{\left\{\eta_{1, i} \leq \ell_{r \oplus 1,1}-x_{1}, \eta_{2, i}+\eta_{3, i}>\ell_{r \oplus 1,2}-x_{2}\right\}} \mid A_{i}\left(r ; x_{1}, x_{2}, x_{3}\right)\right) \\
=\sum_{x_{1}=0}^{\ell_{r \oplus 1,1}} \sum_{x_{2}=0}^{\infty} \sum_{x_{3}=0}^{\infty} Q_{i}\left(r ; x_{1}, x_{2}, x_{3}\right) \\
\quad \times \sum_{\ell_{r}=0}^{\ell_{1,1}-x_{1}} \sum_{c=m_{1}}^{\infty} \sum_{b_{2}=m_{2}}^{\infty} \varphi_{1}\left(b_{1} ; T_{r \oplus 1}\right) \psi\left(c ; x_{3}, T_{r \oplus 1}\right) z_{2}^{x_{2}+b_{2}+c-\ell_{r \oplus 1,2}} \\
\quad \times\left(z_{3}^{x_{1}+x_{3}+b_{1}-c}-z_{1}^{x_{1}+b_{1}-\ell_{r \oplus 1,1}} z_{3}^{\ell_{r \oplus 1,1}+x_{3}-c}\right)
\end{aligned}
$$

and

$$
\begin{aligned}
& \sum_{x \in X} Q_{i}\left(r ; x_{1}, x_{2}, x_{3}\right) \mathrm{E}\left(\left(z_{1}^{x_{1}+\eta_{1, i}-\ell_{r \oplus 1,1}} z_{3}^{x_{3}+\ell_{r \oplus 1,1}-\eta_{3, i}}-Z\left(x_{1}, x_{2}, x_{3}\right)\right.\right. \\
& \left.\quad \times I_{\left\{\eta_{1, i}>\ell_{r \oplus 1,1}-x_{1}, \eta_{2, i}+\eta_{3, i} \leq \ell_{r \oplus 1,2}-x_{2}\right\}} \mid A_{i}\left(r ; x_{1}, x_{2}, x_{3}\right)\right) \\
& =\sum_{x_{1}=0}^{\infty} \sum_{x_{2}=0}^{\ell_{r \oplus 1,2}} \sum_{x_{3}=0}^{\infty} Q_{i}\left(r ; x_{1}, x_{2}, x_{3}\right) z_{1}^{x_{1}-\ell_{r \oplus 1,1}} z_{3}^{x_{3}+\ell_{r \oplus 1,2}} \\
& \quad \times \sum_{b_{1}=m_{3}+1}^{\infty} \sum_{c=0}^{\min \left\{x_{3}, \ell_{r \oplus 1,2}\right\}} \sum_{b_{2}=0}^{\ell_{r \oplus 1,2}-x_{2}-c} \varphi_{1}\left(b_{1} ; T_{r \oplus 1}\right) \varphi_{2}\left(b_{2} ; T_{r \oplus 1}\right) \\
& \times \psi\left(c ; x_{3}, T_{r \oplus 1}\right) z_{1}^{b_{1}}\left(1-z_{2}^{x_{2}+b_{2}+c-\ell_{r \oplus 1,2}}\right) z_{3}^{-c} .
\end{aligned}
$$


Also

$$
\begin{aligned}
& \sum_{x \in X} Q_{i}\left(r ; x_{1}, x_{2}, x_{3}\right) \mathrm{E}\left[\left(z_{3}^{x_{3}+x_{1}+\eta_{1, i}-\eta_{3, i}}-Z\left(x_{1}, x_{2}, x_{3}\right)\right)\right. \\
& \left.\times I_{\left\{\eta_{1, i} \leq \ell_{r \oplus 1,1}-x_{1}, \eta_{2, i}+\eta_{3, i} \leq \ell_{r \oplus 1,2}-x_{2}\right\}} \mid A_{i}\left(r ; x_{1}, x_{2}, x_{3}\right)\right] \\
& =\sum_{x_{1}=0}^{\ell_{r \oplus 1,1}} \sum_{x_{2}=0}^{\ell_{r \oplus 1,2}} \sum_{x_{3}=0}^{\infty} Q_{i}\left(r ; x_{1}, x_{2}, x_{3}\right) \\
& \times \sum_{b_{1}=0}^{\ell_{r \oplus 1,1}-x_{1}} \sum_{c=0}^{m_{4}} \sum_{b_{2}=0}^{\ell_{r \oplus 1,2}-x_{2}-c} \varphi_{1}\left(b_{1} ; T_{r \oplus 1}\right) \varphi_{2}\left(b_{2} ; T_{r \oplus 1}\right) \psi\left(c ; x_{3}, T_{r \oplus 1}\right) \\
& \times\left(z_{3}^{x_{1}+x_{3}+b_{1}-c}-z_{1}^{x_{1}+b_{1}-\ell_{r \oplus 1,1}} z_{2}^{x_{2}+b_{2}+c-\ell_{r \oplus 1,2}} z_{3}^{x_{3}+\ell_{r \oplus 1,1}-c}\right) .
\end{aligned}
$$

The theorem is proved for $\Gamma^{(r \oplus 1)} \in \Gamma^{\mathrm{IV}}$.

The recurrence relations of Theorem 3 allow one to evaluate the probability generating functions $\Psi_{i+1}\left(r ; z_{1}, z_{2}, z_{3}\right), r=1,2, \ldots, n, i=0,1, \ldots$, from a given probability distribution of the vector $\left(\Gamma_{0}, \kappa_{1,0}, \kappa_{2,0}, \kappa_{3,0}\right)$. Note that the probability generating functions converge in the polydisk $D_{0}=\left\{\left(z_{1}, z_{2}, z_{3}\right):\left|z_{i}\right| \leq 1\right\}$.

The following result shows that the probability generating functions converge in the polydisk $D_{\varepsilon}=\left\{\left(z_{1}, z_{2}, z_{3}\right):\left|z_{i}\right| \leq 1+\varepsilon\right\}$ provided that a point $\left(z_{1}, z_{2}, z_{3}\right)$ does not belong to $D_{\varepsilon}$ if $z_{1}$ is a singular point of the function $q_{r, 1}\left(z_{1}\right)$ for some $r$ or if $z_{2}$ is a singular point of the function $q_{r, 2}\left(z_{2}\right)$ for some $r$ if $\Psi_{0}\left(r ; z_{1}, z_{2}, z_{3}\right)$ is chosen appropriately.

Lemma 1. The function $\left(z_{1}, z_{2}, z_{3}\right) \rightarrow\left(z_{1}, z_{2}, p z_{2}+(1-p) z_{3}\right)$ maps the polydisk $D_{\varepsilon}$ into itself if $0<p<1$.

Proof. Consider the polar coordinates $z_{j}=\rho_{j} e^{i u_{j}}, j=1,2,3$. Then $\rho_{j} \leq 1+\varepsilon$ in the polydisk $D_{\varepsilon}$. Hence

$$
\begin{aligned}
\left|p z_{2}+(1-p) z_{3}\right|^{2} & =p^{2} \rho_{2}^{2}+(1-p)^{2} \rho_{3}^{2}+2 p(1-p) \rho_{2} \rho_{3} \cos \left(u_{2}-u_{3}\right) \\
& \leq\left(p \rho_{2}+(1-p) \rho_{3}\right)^{2} \leq(1+\varepsilon)^{2} .
\end{aligned}
$$

Thus $\left(z_{1}, z_{2}, p z_{2}+(1-p) z_{3}\right) \in D_{\varepsilon}$. The lemma is proved.

\section{A sUfFiCiENT CONDITION FOR THE EXISTENCE OF THE STATIONARY DisTRIBUtion}

Let

$$
\ell_{1}=\sum_{r: \Gamma^{(r)} \in \Gamma^{\mathrm{II}} \cup \Gamma^{\mathrm{IV}}} \ell_{r, 1}, \quad \ell_{2}=\sum_{r: \Gamma^{(r)} \in \Gamma^{\mathrm{III}} \cup \Gamma^{\mathrm{IV}}} \ell_{r, 2},
$$

$T=T_{1}+T_{2}+\cdots+T_{n}$, and

$$
\bar{\lambda}_{j}=\lambda_{j} \sum_{x=1}^{\infty} x p_{x}^{(j)} .
$$

Here $\ell_{j}$ means the number of customers coming from the saturation flow $\Pi_{j}^{\text {sat }}$ during the server working period of duration $T$, while $\bar{\lambda}_{j}$ is the intensity of the flow $\Pi_{j}$, since $\sum_{x=1}^{\infty} x p_{x}^{(j)}$ equals the mathematical expectation of the bulk size in the flow $\Pi_{j}$.

The following result contains some restrictions on intensities of the input flows, mean bulk sizes, saturation flows, and sojourn times of the server that are sufficient for the boundedness in time of the expected number of customers in the system. 
Theorem 4. If

$$
\bar{\lambda}_{1} T-\ell_{1}<0, \quad\left(\bar{\lambda}_{1}+\bar{\lambda}_{2}\right) T-\ell_{2}<0,
$$

then the stationary distribution of the Markov chain (2) exists.

Proof. Assume that the stationary distribution does not exist. Then the sequence

$$
\left\{\mathrm{E}\left(\kappa_{1, i}+\kappa_{2, i}+\kappa_{3, i}\right) ; i=0,1, \ldots\right\}
$$

increases unboundedly for any given initial distribution. Choose a polydisk $D_{\varepsilon}$ that does not contain the points $\left(z_{1}, z_{2}, z_{3}\right)$ such that $z_{1}$ is a singular point of the function $q_{r^{\prime}, 1}\left(z_{1}\right)$ or $z_{2}$ is a singular point of the function $q_{r^{\prime}, 2}\left(z_{2}\right)$ for some $r^{\prime}=1,2, \ldots, n$. Then we choose the initial probability distribution such that the functions $\Psi_{0}\left(r ; z_{1}, z_{2}, z_{3}\right), r=1,2, \ldots, n$, converge in the polydisk $D_{\varepsilon}$. Now we show that the sequence $\left\{\mathrm{E}\left(\kappa_{1, i}+\kappa_{2, i}+\kappa_{3, i}\right)\right.$; $i=0,1, \ldots\}$ is bounded for this initial distribution.

Let $\left(z^{*}, 1,1\right) \in D_{\varepsilon}$ be a point such that $1<z^{*}<1+\varepsilon$. We prove that the sequence $\Psi_{i}\left(r ; z^{*}, 1,1\right), i=0,1, \ldots$, is bounded for each $r$. Theorem 3 implies that

$$
\Psi_{i+1}\left(r \oplus 1 ; z^{*}, 1,1\right)=q_{r \oplus 1,1}\left(z^{*}\right) \Psi_{i}\left(r ; z^{*}, 1,1\right)
$$

for $\Gamma^{(r \oplus 1)} \in \Gamma^{\mathrm{I}} \cup \Gamma^{\mathrm{III}}$, while

$$
\begin{aligned}
\Psi_{i+1}\left(r \oplus 1 ; z^{*}, 1,1\right)= & \left(z^{*}\right)^{-\ell_{r \oplus 1,1}} q_{r \oplus 1,1}\left(z^{*}\right) \Psi_{i}\left(r ; z^{*}, 1,1\right) \\
& +\sum_{x_{1}=0}^{\ell_{r \oplus 1,1}} \sum_{x_{2}=0}^{\infty} \sum_{x_{3}=0}^{\infty} Q_{i}\left(r ; x_{1}, x_{2}, x_{3}\right) \\
& \times \sum_{b_{1}=0}^{\ell_{r \oplus 1,1}-x_{1}}\left(1-\left(z^{*}\right)^{x_{1}+b_{1}-\ell_{r \oplus 1,1}}\right) \varphi_{1}\left(b_{1} ; T_{r \oplus 1}\right)
\end{aligned}
$$

for $\Gamma^{(r \oplus 1)} \in \Gamma^{\mathrm{II}} \cup \Gamma^{\mathrm{IV}}$. Since $Q_{i}\left(r ; x_{1}, x_{2}, x_{3}\right)$ is a probability distribution,

$$
\sum_{x_{2}=0}^{\infty} \sum_{x_{3}=0}^{\infty} Q_{i}\left(r ; x_{1}, x_{2}, x_{3}\right) \leq 1
$$

whence

$$
\begin{aligned}
\Psi_{i+1}\left(r \oplus 1 ; z^{*}, 1,1\right) \leq & \left(z^{*}\right)^{-\ell_{r \oplus 1,1}} q_{r \oplus 1,1}\left(z^{*}\right) \Psi_{i}\left(r ; z^{*}, 1,1\right) \\
& +\sum_{x_{1}=0}^{\ell_{r \oplus 1,1}} \sum_{b_{1}=0}^{\ell_{r \oplus 1,1}-x_{1}}\left(1-\left(z^{*}\right)^{x_{1}+b_{1}-\ell_{r \oplus 1,1}}\right) \varphi_{1}\left(b_{1} ; T_{r \oplus 1}\right) .
\end{aligned}
$$

We find from relations (17) and (18) that

$$
\sum_{r=1}^{n} \Psi_{i+n}\left(r ; z^{*}, 1,1\right) \leq\left(\prod_{r=1}^{n} q_{r, 1}\left(z^{*}\right)\right)\left(z^{*}\right)^{-\ell_{1}}\left(\sum_{r=1}^{n} \Psi_{i}\left(r ; z^{*}, 1,1\right)\right)+B\left(z^{*}\right) .
$$

The inequality $\bar{\lambda}_{1} T-\ell_{1}<0$ yields

$$
\prod_{r=1}^{n} q_{r, 1}\left(z^{*}\right)\left(z^{*}\right)^{-\ell_{1}}<1
$$

The sequence

$$
\begin{gathered}
M_{0}=\sum_{r=1}^{n} \Psi_{0}\left(r ; z^{*}, 1,1\right), \ldots, M_{n-1}=\sum_{r=1}^{n} \Psi_{n-1}\left(r ; z^{*}, 1,1\right), \\
M_{i+n}=M_{i} \prod_{r=1}^{n} q_{r, 1}\left(z^{*}\right)\left(z^{*}\right)^{-\ell_{1}}+B\left(z^{*}\right), \quad i=0,1, \ldots,
\end{gathered}
$$


converges and thus is bounded. Moreover,

$$
\sum_{r=1}^{n} \Psi_{i}\left(r ; z^{*}, 1,1\right) \leq M_{i}, \quad i=0,1, \ldots
$$

Hence $\Psi_{i}\left(r ; z^{*}, 1,1\right), i=0,1, \ldots$, are bounded by a constant $M^{*}$ for all $r$.

Substituting $z_{1}=z_{2}=z_{3}=z^{*}$ in the recurrence relations of Theorem 3, we get

$$
\Psi_{i+1}\left(r \oplus 1 ; z^{*}, z^{*}, z^{*}\right)=q_{r \oplus 1,1}\left(z^{*}\right) q_{r \oplus 1,2}\left(z^{*}\right) \Psi_{i}\left(r ; z^{*}, z^{*}, z^{*}\right)
$$

for $\Gamma^{(r \oplus 1)} \in \Gamma^{\mathrm{I}} \cup \Gamma^{\mathrm{II}}$, while for $\Gamma^{(r \oplus 1)} \in \Gamma^{\mathrm{III}} \cup \Gamma^{\mathrm{IV}}$,

$$
\begin{aligned}
& \Psi_{i+1}\left(r \oplus 1 ; z^{*}, 1,1\right) \\
& =\left(z^{*}\right)^{-\ell_{r \oplus 1,2}} q_{r \oplus 1,1}\left(z^{*}\right) q_{r \oplus 1,2}\left(z^{*}\right) \Psi_{i}\left(r ; z^{*}, z^{*}, z^{*}\right) \\
& +\left(z^{*}\right)^{-\ell_{r \oplus 1,2}} q_{r \oplus 1,1}\left(z^{*}\right) q_{r \oplus 1,2}\left(z^{*}\right) \\
& \quad \times \sum_{x_{1}=0}^{\infty} \sum_{x_{2}=0}^{\ell_{r \oplus 1,2}} \sum_{x_{3}=0}^{\ell_{r \oplus 1,2}-x_{2}} Q_{i}\left(r ; x_{1}, x_{2}, x_{3}\right) \\
& \quad \times \sum_{c=0}^{m_{4}} \sum_{b_{2}=0}^{\ell_{r \oplus 1,2}-x_{2}-c}\left(z^{*}\right)^{x_{1}+x_{3}-c}\left(1-\left(z^{*}\right)^{x_{2}+b_{2}+c-\ell_{r \oplus 1,2}}\right) \\
& \times \varphi_{2}\left(b_{2} ; T_{r \oplus 1}\right) \psi\left(c ; x_{3}, T_{r \oplus 1}\right) .
\end{aligned}
$$

It is easy to see that

$$
\begin{aligned}
& \sum_{x_{1}=0}^{\infty} \sum_{x_{2}=0}^{\ell_{r \oplus 1,2}} \sum_{x_{3}=0}^{\ell_{r \oplus 1,2}-x_{2}} Q_{i}\left(r ; x_{1}, x_{2}, x_{3}\right) \\
& \times \sum_{c=0}^{m_{4}} \sum_{b_{2}=0}^{\ell_{r \oplus 1,2}-x_{2}-c}\left(z^{*}\right)^{x_{1}+x_{3}-c}\left(1-\left(z^{*}\right)^{x_{2}+b_{2}+c-\ell_{r \oplus 1,2}}\right) \varphi_{2}\left(b_{2} ; T_{r \oplus 1}\right) \psi\left(c ; x_{3}, T_{r \oplus 1}\right) \\
& \leq\left(\operatorname { m a x } _ { 0 \leq x _ { 2 } + x _ { 3 } \leq \ell _ { r } \oplus 1 , 2 } \left\{\sum_{c=0}^{m_{4}} \sum_{b_{2}=0}^{\ell_{r \oplus 1,2}-x_{2}-c}\left(z^{*}\right)^{x_{3}-c}\right.\right. \\
& \left.\left.\times\left(1-\left(z^{*}\right)^{x_{2}+b_{2}+c-\ell_{r \oplus 1,2}}\right) \varphi_{2}\left(b_{2} ; T_{r \oplus 1}\right) \psi\left(c ; x_{3}, T_{r \oplus 1}\right)\right\}\right) \\
& \times \sum_{x_{1}=0}^{\infty} \sum_{x_{2}=0}^{\ell_{r \oplus 1,2}} \sum_{x_{3}=0}^{\ell_{r \oplus 1,2}-x_{2}}\left(z^{*}\right)^{x_{1}} Q_{i}\left(r ; x_{1}, x_{2}, x_{3}\right) .
\end{aligned}
$$

At the same time,

$$
\begin{aligned}
\sum_{x_{1}=0}^{\infty} \sum_{x_{2}=0}^{\ell_{r \oplus 1,2}} \sum_{x_{3}=0}^{\ell_{r \oplus 1,2}-x_{2}}\left(z^{*}\right)^{x_{1}} Q_{i}\left(r ; x_{1}, x_{2}, x_{3}\right) & \leq \sum_{x_{1}=0}^{\infty} \sum_{x_{2}=0}^{\infty} \sum_{x_{3}=0}^{\infty}\left(z^{*}\right)^{x_{1}} Q_{i}\left(r ; x_{1}, x_{2}, x_{3}\right) \\
& =\Psi_{i}\left(r ; z^{*}, 1,1\right) .
\end{aligned}
$$

Since $\Psi_{i}\left(r ; z^{*}, 1,1\right)<M^{*}$, the second term on the right hand side of equation (20) is bounded from above by another constant that does not depend on $i$. Thus equations (19) and (20) imply

$$
\sum_{r=1}^{n} \Psi_{i+n}\left(r ; z^{*}, z^{*}, z^{*}\right) \leq\left(\prod_{r=1}^{n} q_{r, 1}\left(z^{*}\right) q_{r, 2}\left(z^{*}\right)\right)\left(z^{*}\right)^{-\ell_{2}}\left(\sum_{r=1}^{n} \Psi_{i}\left(r ; z^{*}, z^{*}, z^{*}\right)\right)+B_{1}\left(z^{*}\right) .
$$


By inequality $\left(\bar{\lambda}_{1}+\bar{\lambda}_{2}\right) T-\ell_{2}<0$, we have

$$
\prod_{r=1}^{n} q_{r, 1}\left(z^{*}\right) q_{r, 2}\left(z^{*}\right)\left(z^{*}\right)^{-\ell_{2}}<1 .
$$

The sequence

$$
\begin{gathered}
M_{0}^{\prime}=\sum_{r=1}^{n} \Psi_{0}\left(r ; z^{*}, z^{*}, z^{*}\right), \ldots, M_{n-1}^{\prime}=\sum_{r=1}^{n} \Psi_{n-1}\left(r ; z^{*}, z^{*}, z^{*}\right), \\
M_{i+n}^{\prime}=M_{i}^{\prime}\left(\prod_{r=1}^{n} q_{r, 1}\left(z^{*}\right) q_{r, 2}\left(z^{*}\right)\right)\left(z^{*}\right)^{-\ell_{2}}+B_{1}\left(z^{*}\right), \quad i=0,1, \ldots,
\end{gathered}
$$

converges and thus is bounded. Moreover,

$$
\sum_{r=1}^{n} \Psi_{i}\left(r ; z^{*}, z^{*}, z^{*}\right) \leq M_{i}^{\prime}, \quad i=0,1, \ldots
$$

Therefore the sequence $\Psi_{i}\left(r ; z^{*}, z^{*}, z^{*}\right), i=0,1, \ldots$, is bounded by a constant $M^{* *}$ for all $r$. According to Cauchy's theorem, the expectation

$$
\mathrm{E}\left(\kappa_{1, i}+\kappa_{2, i}+\kappa_{3, i}\right)=\frac{1}{2 \pi \sqrt{-1}} \int_{|z-1|=\rho}(z-1)^{-2} \sum_{r=1}^{n} \Psi_{i}(r ; z, z, z) d z
$$

is bounded from above and the bound does not depend on $\rho$ and $M^{* *}$. This proves that the mathematical expectation of the total number of customers in the system is bounded. The theorem is proved.

It would be instructive to analyze the sufficient condition in Theorem 4 . The first inequality ensures that the queue $O_{1}$ remains bounded in mean as the time increases. The intensity of the output flow from the queue $O_{1}$ is equal to the intensity of the input flow $\Pi_{1}$. The second inequality means that the cumulative input intensity for the queue $O_{2}$ is equal to $\bar{\lambda}_{1}+\bar{\lambda}_{2}$ and does not depend on the probabilities $p_{r}, r=1,2, \ldots, n$. The interpretation of the second inequality is that the system is capable of serving "in time" all the customers coming to the queue $\mathrm{O}_{2}$.

\section{BIBLIOGRAPHY}

1. M. A. Fedotkin, Optimal control for conflicting flows and marked point processes with an isolated discrete component. I, Litovsk. Mat. Sb. 28 (1988), no. 4, 783-794. (Russian) MR.987873 (90e:90056)

2. M. A. Fedotkin, Queueing processes and control systems, Mathematical problems in cybernetics 6 (1996), Nauka, Moscow, pp. 51-70. (Russian) MR.1856119

3. M. A. Fedotkin, A nonlocal method for determining control random processes, Mathematical problems in cybernetics 7 (1998), Nauka, Moscow, pp. 333-344. (Russian) MR1843047

4. A. V. Zorin and M. A. Fedotkin, Optimization of control of doubly stochastic nonordinary flows in time-sharing systems, Avtomat. i Telemekh. 66 (2005), no. 7, 102-111; English transl. in Autom. Remote Control 66 (2005), no. 7, 1115-1124. MR2167835

Department of Applied Probability Theory, Faculty for Computational Mathematics and Cybernetics, LobachevskiI State University of NizhniI Novgorod - National Research University, Gagarin Avenue 23, Nizhni Novgorod 603950, Russia

E-mail address: zoav1602@gmail.com

Received 13/OCT/2010

Originally published in Russian

Translated by N. SEMENOV 